\title{
Music Genre Classification and Recommendation
}

\section{Prof. Rahul Ghode ${ }^{1}$, Pranav Navale ${ }^{2}$, Mayur Jadhav², Anirudha Chippa ${ }^{2}$, Minal Bhandare ${ }^{2}$}

${ }^{1}$ Professor at Information Technology Department, Dhole Patil College of Engineering, Wagholi, Pune, Maharashtra, India

2B. E. Scholar, Information Technology Department, Dhole Patil College of Engineering, Wagholi, Pune, Maharashtra, India

Article Info

Volume 7, Issue 6

Page Number: 298-301

\section{Publication Issue :}

November-December-2021

\section{Article History}

Accepted : 05 Dec 2021

Published : 20 Dec 2021

\section{ABSTRACT}

There are various sorts to group the music. Classes are for the most part various classifications wherein music is partitioned. In this day and age as music industry develops quickly, there are various kinds of music sorts made. It is essential to classify the music into these classifications, yet it is mind boggling task. In past times this is done physically and prerequisite for programmed framework for type grouping emerges. As a rule, AI techniques are utilized to group music types and profound learning strategy is utilized to prepare the model yet in this undertaking, we will utilize neural organization strategies for the characterization.

Keywords : Artificial Intelligence, Genre, Neural organization, Harcascade, Hyper-plane, Support Vector Machines

\section{INTRODUCTION}

AI has become extremely famous as of late. Contingent upon the kind of utilization and the dataset accessible, particular sorts of AI methods are more proper than others for various applications. The primary sorts of learning calculations incorporate regulated learning, solo learning, semi-administered learning, and support learning. A neural organization $(\mathrm{NN})$ is a strategy of AI that is by and large viable at extricating basic elements from complex datasets and inferring a capacity or model that communicates those elements. The NN uses a preparation dataset to initially prepare a model. After the model is prepared, the NN would then be able to be applied to new or beforehand inconspicuous informative elements and order the information dependent on the recently prepared model.

\section{LITERATURE SURVEY}

Miao Jiang, Ziyi Yang, Chen Zhao, "An RNN-based music recommendation system." [1] In the very recent years, development of music recommendation system has been a more heated problem due to a higher level of digital songs consumption and the advancement of machine learning techniques. Some traditional approaches such as collaborator filtering, has been widely used in recommendation systems, have helped music recommendation system to give music listeners a quick access to the music. However, collaborative filtering or model-based algorithm have 
limitations in giving a better result with the ignorance of combination factor of lyrics and genre.

In our paper, we will propose an improved algorithm based on deep neural network on measure similarity between different songs. The proposed method will make it possible that it could make recommendations in a large system to make comparisons by "understand" the content of songs.

Sushmita G. Kamble and Asso. Prof. A. H. Kulkarni, "Facial Expression Based Music Player." [2] Conventional method of playing music depending upon the mood of a person requires human interaction. Migrating to the computer vision technology will enable automation of such system. To achieve this goal, an algorithm is used to classify the human expressions and play a music track as according to the present emotion detected.

Jie Liu, Liang Jia, "The Application of Computer Music Technology in Music Education" [3] With the rapid development of Internet technology, computers are widely used in all walks of life, and among them, computer music technology has become one of the important tools in modern music education. Analysis of the traditional music teaching mode can be found, most of the time is limited to the teacher performance, student imitation, however, for the new era of music education, the combination of computer technology and music knowledge has brought great convenience for the development of music education

George Tzanetakis, Student Member, IEEE, and Perry Cook, Member, IEEE, "Musical Genre Classification of Audio Signals. "[4] Three feature sets for representing timbral texture, rhythmic content and pitch content are proposed. Features is investigated by training statistical pattern recognition classifiers using real-world audio collections.
Liang Stamatakis, Jie Liu, "An exploration of the application of computer music production software in music composition" [5] The gradual implementation of computer music production software within the current stage of music composition and traditional composition methods for integration is important for the creation of excellent musical works with unique styles and novel ideas.

Federico Simonetta, Stavros Ntalampiras, "Multimodal Music Information Processing and Retrieval: Survey and Future Challenges" [6] Towards improving the performance in various music information processing tasks, recent studies exploit different modalities able to capture diverse aspects of music. Such modalities include audio recordings, symbolic music scores, mid-level representations, motion and gestural data, video recordings, editorial or cultural tags, lyrics and album cover arts.

\section{PROPOSED SYSTEM}

The programmed examination and comprehension of music by the PC is the additional opportunity in the field of music data retrieval. First, we login in the framework. In catch stage we catch the face or picture in the camera. Here we utilize a camera for face identification. The principal objective of face identification procedure is to distinguish the face which is cheerful or furious. In the following square which is Affix API. An application programming interface is registering interface which characterizes cooperation between numerous products. Programming interface permits two applications to converse with one another. It is connection between catch stage and fundamental stage.

In information stockpiling stage we as of now store the picture which are cheerful, dismal or furious. This information and approaching information from catch stage are analyze in the fundamental stage and naturally played tunes for this specific stage which is shown in a presentation stage. 
Neural Network is utilized to order music types.

A convolutional neural organization $(\mathrm{CNN})$ is a sort of impartial organization that is planned to process multi-dimensional clusters like pictures. Pictures of spectrograms produced from time-cuts of tunes as the contribution to a neural organization to group the melodies into their separate melodic classifications.

\section{SYSTEM ARCHITECTURE}

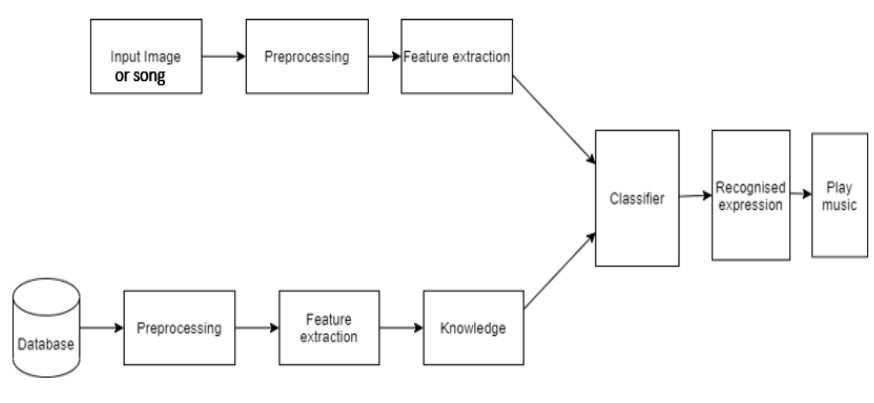

Fig. System Architecture

\section{ALGORITHM}

CNN stands for Convolutional Neural Networks, which are specialized for image and video recognition applications. Image recognition, object detection, and segmentation are among of the most common image analysis tasks that $\mathrm{CNN}$ is employed for.

Convolutional Neural Networks have four different sorts of layers:

1) Convolutional Layer: Each input neuron in a conventional neural network is linked to the next hidden layer. Only a small portion of the input layer neurons connect to the hidden layer neurons in CNN.

2) Pooling Layer: The pooling layer is used to minimize the feature map's dimensionality. Inside the CNN's hidden layer, there will be several activation and pooling layers.

3) Flatten: Flattening is the process of transforming data into a one-dimensional array for use in the next layer. To construct a single lengthy feature vector, we flatten the output of the convolutional layers.

4) Fully Connected Layers: Fully Connected Layers are the network's final layers. The output from the final Pooling or Convolutional Layer, which is flattened and then fed into the fully connected layer, is the input to the fully connected layer.

\section{Support Vector Machines:}

It is supervised machine learning algorithm. It is used for classification ad regression. In this algorithm, features are extracted from input ad plotted it on ndimensional space where $\mathrm{n}$ is number of features i.e., each coordinate is value of particular feature.

SVM works as follow:

1) Plotting features on $n$-dimensional space

2) Identifying right hyper-plane

3) Classifying the data according to that hyperplane

\section{Harcascade:}

The algorithm can be explained in four stages:

1. Calculating Haar Features

2. Creating Integral Images

3. Using Adaboost

4. Implementing Cascading Classifiers

It's important to remember that this algorithm requires a lot of positive images of faces and negative images of non-faces to train the classifier, similar to other machine learning models.

Calculating Haar Features_

The first step is to collect the Haar features. A Haar feature is essentially calculations that are performed on adjacent rectangular regions at a specific location in a detection window. The calculation involves summing the pixel intensities in each region and calculating the differences between the sums.

\section{CONCLUSION}

We are Developing a framework to perceive client feeling dependent on look utilizing Python. We Integrate the python code into the web administration and play the music dependent on the look like cheerful, miserable or impartial. It is generally excellent amusement for the clients. Feeling 
acknowledgment utilizing looks is one of the significant subjects of exploration and has accumulated a lot of consideration before. It very well may be seen that the issue of feeling acknowledgment with the assistance of picture handling calculations has been expanding step by step. Specialists are consistently chipping away at ways of settling this by the utilization of various types of highlights and picture handling strategies.

\section{REFERENCES}

[1]. S L Happy and Aurobinda Routray, "Automatic Facial Expression Recognition using Features of salient Facial Patches," in IEEE Trans. On Affective Computing, January-March 2015, pp. 1-12.

[2]. Hafeez Kabani, Sharik Khan, Omar Khan and Shabana Tadvi, "Emotion based Music Player," Int. J. of Eng. Research and General Sci., Vol. 3, Issue 1, pp. 750-756, January-February 2015.

[3]. Li Siquan, Zhang Xuanxiong, "Research on Facial Expression Recognition Based on Convolutional Neural Networks", Journal of Software, 2018, v.17; No.183 (01): 32-35.

[4]. Hou Yuqingyang, Quan Jicheng, Wang Hongwei, "Overview of the development of deep learning", Ship Electronic Engineering, 2017, 4: 5-9.

[5]. Liu Sijia, Chen Zhikun, Wang Fubin, "Multiangle face recognition based on convolutional neural network", Journal of North China University of Technology (Natural Science Edition), 2019, 41 (4): 103-108.

[6]. Li Huihui, "Research on facial expression recognition based on cognitive machine learning", Guangzhou: South China University of Technology, 2019.

[7]. Li Yong, Lin Xiaozhu, Jiang Mengying, "Facial expression recognition based on crossconnection", LeNet-5 network J], Journal of Automation, 2018,44 (1): 176-182.
[8]. Yao L S, Xu G M, Zhap F, "Facial Expression Recognition Based on CNN Local Feature Fusion", Laser and Optoelectronics Progress, 2020, 57(03): 032501.

[9]. Xie S, Hu H., "Facial expression recognition with FRR-CNN", Electronics Letters, 2017, 53 (4): 235-237.

[10]. Nikki Pelchat, Craig M Gelowitz, "Neural Network Music Genre Classification”, 2019.

[11]. Vishnupriya S, K. Meenakshi, "Automatic Music Genre Classification using Convolution Neural Network", International Conference on Computer Communication and Informatics (ICCCI -2017), Coimbatore, 2018.

[12]. George Tzanetakis and Perry Cook, "Musical Genre Classification of Audio Signals", IEEE Transactions on Speech and Audio Processing, VOL. 10, NO. 5, JULY 2002.

[13]. Rajeeva Shreedhara Bhat, Rohit B. R., Mamatha K. R., "Music Genre Classification", SSRG International Journal of Communication and Media Science (SSRG-IJCMS) - Volume 7 Issue 1 - Jan - April 2020.

[14]. Kaichun K. Chang, Jyh-Shing Roger Jang, Costas S. Iliopoulos, "Music Genre Classification Via Compressive Sampling", 11th International Society for Music Information Retrieval Conference.

[15]. Beici Liang, Minwei Gu, "Music Genre Classification Using Transfer Learning", IEEE Conference on Multimedia Information Processing and Retrieval (MIPR), 2020.

\section{Cite this article as :}

Prof. Rahul Ghode, Pranav Navale, Mayur Jadhav, Anirudha Chippa, Minal Bhandare, "Music Genre Classification and Recommendation", International Journal of Scientific Research in Computer Science, Engineering and Information Technology (IJSRCSEIT), ISSN : 2456-3307, Volume 7 Issue 6, pp. 298-301, November-December 2021. Available at doi : https://doi.org/10.32628/CSEIT217684 Journal URL : https://ijsrcseit.com/CSEIT217684 